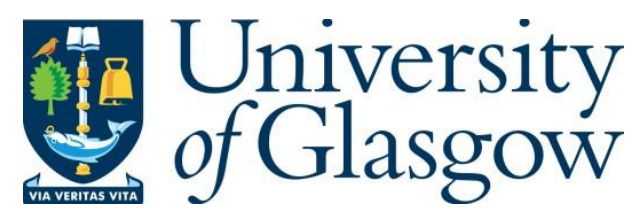

Wei, Y., Zahid, A., Heidari, H., Imran, M. and Abbasi, Q. H. (2019) A Compact NonInvasive WearableVital Signal Monitoring System. In: IEEE Asia Pacific Conference on Postgraduate Research in Microelectronics and Electronics (PrimeAsia 2018), Chengdu, China, 26-30 Oct 2018, pp. 55-59. ISBN 9781538695913.

There may be differences between this version and the published version. You are advised to consult the publisher's version if you wish to cite from it.

$\underline{\text { http://eprints.gla.ac.uk/168790/ }}$

Deposited on: 13 September 2018

Enlighten - Research publications by members of the University of Glasgow http://eprints.gla.ac.uk 


\title{
A compact Non-Invasive WearableVital Signal Monitoring System
}

\author{
Yuan Wei ${ }^{1}$, Adnan Zahid ${ }^{2}$, Hadi Heidari², Muhammad Imran $^{2}$, Qammer H. Abbasi ${ }^{2}$ \\ ${ }^{1}$ University of Electronic Science and Technology of China, Chengdu, China \\ ${ }^{2}$ School Electronics and Nanoscale Engineering, University of Glasgow, G12 8QQ, Glasgow, UK \\ Email: 2168586W@ @student.gla.ac.uk, a.zahid.1@research.gla.ac.uk,Hadi.Heidari@glasgow.ac.uk, \\ Muhammad.Imran@glasgow.ac.uk, Qammer.Abbasi@glasgow.ac.uk
}

\begin{abstract}
On-body vital signal detection and communication is a popular option for applications especially health-care. This paper proposes, simulation design and development of a wearable compact antenna, which provide an alternative solution to on-body vital signals such as breathing and heart rate signal. The vital signals were measured by connecting proposed antenna to the chest using vector network analyzer and feasibility of this new method was verified by comparing the obtained vital signals to the commercial devices and testified the methodology by using Bland Altman analysis. The results obtained shows the $4.8 \%$ and $6.4 \%$ of the respiration result and heart rate result are out of $95 \%$ confidence interval.
\end{abstract}

Index terms: On-body, monitoring, antenna, Bland-Altman analysis, wearable

\section{INTRODUCTION}

In the recent years, most industrialized nations are facing cost complications in providing long-term health care and wellbeing service. This is mainly due to the increasing elderly population, which is expected to reach 2 billion by the half of 21 century. The increase of age-related chronic diseases, such as congestive heart failure, chronic obstructive pulmonary disease, arthritis, osteoporosis, and dementia, also translates into a tremendous strain on various health-care services. [1] Therefore, newly developed diagnosis technologies have born to face not only this challenge but also an opportunity. Non-invasive sensors have raised people's attention since it offers maximum convenience to the patients. The sensor network has evolved to wearable and integrated into the living environment without affecting a person's daily patterns. These promising sensors can change healthcare from snapshot measurements of physiological parameters to continuous monitoring enabling clinicians to provide guidance daily, motivating the solution based on single or combination of sensors can detect the vital signals, such as heart rate, breathing rate and heart rate variability etc [2].

Taking the heart rate and breathing rate monitoring system as an example, the mainstream method is using ECG device to measure the heart beat and the breathing rate can be accessed by using a breathing belt such as EG05000, the commercial ECG module. It can also be achieved by ECG signal using the algorithm [2]. However, the sizes of existing devices is still inconvenient for patients to wear for a long period. If a compact non-invasive sensor can measure both the respiration signal and heartbeat signal simultaneously with acceptable accuracy compared to the mainstream devices like ECG module and breathing belt, then this newly developed technology can increase the convenience of the users such as the patient with heart disease and the athletes [2].

Doppler radar is such a competent antenna for vital signal monitoring and it is a promising tool for heart rate and breathing rate monitoring. A tiny radar can be attached to the chest of patients, which offer extreme mobility compared with ECG device which needs to connect at least two electrodes and numbers of wires. Besides, an extradentary features of Doppler radar vital signal monitoring is that the Doppler radar measures the mechanical movement of the heart [2]. Usually, diseases such as ardiopathy affect the mechanical movement of the heart, which are not apparent to diagnose in electrical activity monitoring. If an antenna can monitor on-body vital signal at a wideband of frequency including $2.45 \mathrm{GHz}$ which is a common frequency in the commercial field and meanwhile equip acceptable accurate comparing to the gold standard method, the efficiency will increase tremendously [2].

In this paper, a brief review related to vital signal monitoring will be discussed. After this, a simulation design and development of a wearable compact antenna is proposed. This antenna has provided an alternative solution to on-body vital signal such as breathing signal and heart rate signal based on the principle of Doppler radar. Besides, a human body modelling has been proposed to evaluate the performance of designed antenna for on-body signal measurement.

After the antenna part, specific hardware fabrication is shown. The vital signal was measured by attaching proposed antenna to the chest using vector network analyzer (VNA), and the obtained signals were separated and dealt with some digital signal process techniques and algorithm. Finally, the feasibility was verified by comparing the obtained vital signals to the commercial devices and testified the methodology by using Bland Altman analysis. 


\section{ANTENNA DESIGN AND SIMULATION}

\section{A. THE ANTENNA DESIGNING}

The desired antenna is designed for detection using VNA device. A small and low-profile antenna is needed with low mutual influence between antenna and body for high radiation efficiency and low specific absorption rate(SAR) with some additional parameters [3]. At the beginning of the experiment, the antenna is considered working at a ultrawide band frequency range for this reason an Ultra-wide band Coplanar Waveguide antenna (CPW) was moderated from [4]. In these experiment, a wideband $\mathrm{CPW}$ monopole antenna without a ground plane was applied. The topology and reflection coefficient(vacuum) is shown in Fig. 1 and Fig. 2

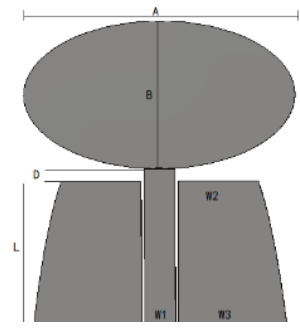

Fig. 1. Topology of CPW antenna $A=44 \mathrm{~mm}, \mathrm{~B}=24 \mathrm{~mm}, \mathrm{G}=0.5 \mathrm{~mm}, \mathrm{~W} 1=5$ $\mathrm{mm}, \mathrm{W} 2=13 \mathrm{~mm}, \mathrm{D}=2 \mathrm{~mm}, \mathrm{~W} 3=17.5 \mathrm{~mm}$ and $\mathrm{L}=25 \mathrm{~mm}$.

\section{B. HUMAN BODY MODELLING}

The antenna should be attached to the human chest, which means a 3D complex and 3 layers chest model will be developed using tissue properties given by [5]. To build the human tissues the permittivity, conductivity and magnetic permeability should be specified. The following graph shows these characteristics. Meanwhile, the thickness of each adipose is also a factor to be evaluated, in this experiment the thickness of skin, adipose and muscle are $4 \mathrm{~mm}, 15 \mathrm{~mm}$ and $25 \mathrm{~mm}$ respectively.

\begin{tabular}{|c|c|c|c|}
\hline & muscle & skin & Adipose \\
\hline $\begin{array}{c}\text { Relative } \\
\text { Permittivity }\end{array}$ & 70 & 50 & 1 \\
\hline Conductivity(S/m) & 1 & 1 & 0.1 \\
\hline $\begin{array}{c}\text { Relative Mag. } \\
\text { Permeability }\end{array}$ & 1 & 1 & 1 \\
\hline
\end{tabular}
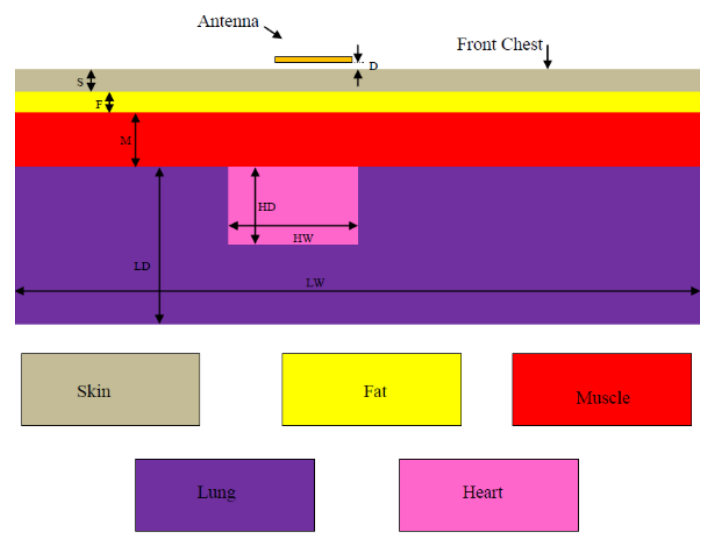

Fig. 2. The $3 \mathrm{D}$ chest model, $\mathrm{S}=4 \mathrm{~mm}, \mathrm{~F}=15 \mathrm{~mm}$ and $\mathrm{M}=25 \mathrm{~mm}$

\section{SIMULATION}

The following figure show the simulated return loss at 2.45 $\mathrm{GHz}$ is nearly $-20 \mathrm{~dB}$, while the measured one is $-15 \mathrm{~dB}$. The difference is around $5 \mathrm{~dB}$.

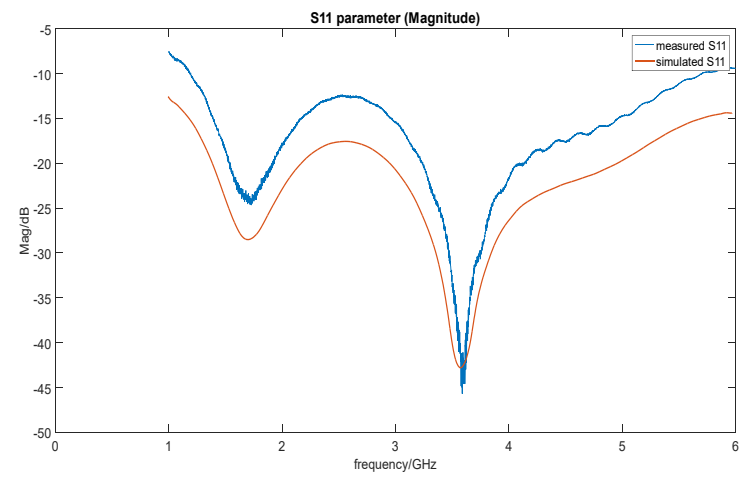

Fig. 3. S11 comparison with measured S11 using VNA

\section{RADIATION PATTERN}

Table II shows the parameters of radiation pattern in vacuum and $3 \mathrm{D}$ chest model. The vacuum radiation pattern showing reveals the main lobe magnitude is $2.91 \mathrm{dBi}$ and the main lobe direction is -1.0 degree with the angular width $(3 \mathrm{~dB})$ $78.7^{\circ}$. The main direction travels along the vertical axis. For the $\mathrm{H}$-plane radiation pattern, there is least radiation magnitude in $0^{\circ}$ and $180^{\circ}$ which means there are few radiations in the vertical axis. Comparing to the radiation pattern in vacuum where the main lobe is located near $0^{\circ}$ and $180^{\circ}$, most of the EM wave travels along the body surface.

\section{TABLE I. RADIATION PATTERN PARAMETERS}

\begin{tabular}{|c|l|l|l|l|l|}
\hline $\begin{array}{c}\text { Simulated } \\
\text { environme } \\
\text { nt }\end{array}$ & $\begin{array}{c}\text { Freque } \\
\text { ncy } \\
(\mathbf{G H z})\end{array}$ & $\begin{array}{c}\text { Main lobe } \\
\text { magnitud } \\
\boldsymbol{e} \\
(\mathbf{d B})\end{array}$ & $\begin{array}{c}\text { Main lobe } \\
\text { direction } \\
()^{\circ}\end{array}$ & $\begin{array}{c}\text { Angular } \\
\text { wide } \\
(\%)\end{array}$ & $\begin{array}{c}\text { Side } \\
\text { lobe } \\
\text { level } \\
(\text { dB })\end{array}$ \\
\hline $\begin{array}{c}\text { H Plane } \\
\text { (vacuum) }\end{array}$ & 2.45 & 2.91 & 1.0 & 78.7 & $/$ \\
\hline $\begin{array}{c}\text { E Plane } \\
\text { (vacuum) }\end{array}$ & 2.45 & 2.91 & $/$ & $/$ & $/$ \\
\hline $\begin{array}{c}\text { H plane } \\
\text { (3D chest } \\
\text { model) }\end{array}$ & 2.45 & 2.29 & 88.0 & 67.5 & -2.5 \\
\hline $\begin{array}{c}\text { E plane } \\
\text { (3D chest } \\
\text { model) }\end{array}$ & 2.45 & 4.43 & 315 & 142.8 & -4.5 \\
\hline
\end{tabular}

III. NON-INVASIVE VITAL SIGNAL MEASUREMENTS

\section{A. EXPERIMENTAL SETUP}

The basic components to monitor the on-body vital signals (breathing signal and heartrate signal) consists of a VNA (Vector Network Analyzer) and the designed antenna. A commercial ECG device BMD101 was used as a referenced for heart rate and breathing rate. This module with two $\mathrm{Ag} / \mathrm{AgCl}$ electrodes was attaching to the upper left chest to get the desired signal and the signal was transmitted to the PC by Bluetooth for further analysis. The antenna was attached to the front left chest which 
is a recommended position mentioned in [12] and connected to the VNA. The breathing rate was calculated using the commercial algorithm and the ECG signal was calculated using 8 window averaging method.

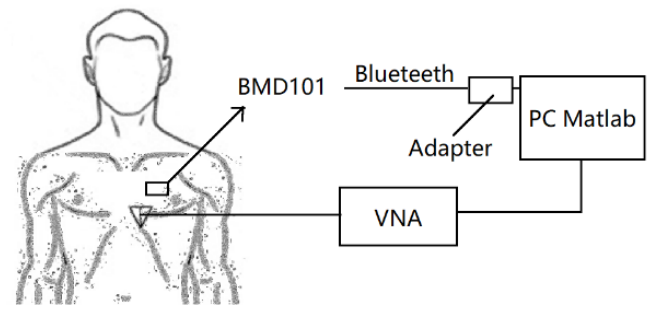

Fig. 4. Gerneral experiment setup showing the VNA and BMD101

TABELE II. EXPERIMENTAL PARAMETER SET UP

\begin{tabular}{|l|l|l|}
\hline Antenna & & CPW antenna \\
\hline Sampling rate & & 30 sample/sec \\
\hline Power & & $-10 \mathrm{dBm}$ \\
\hline
\end{tabular}

\section{B. BREATHING RATE MEASUREMENT}

The obtained on-body signal mainly contains heartrate signal breathing signal and high-frequency noise. For the breathing signal, periodical change is quite dominating (more than 10 times than the heartrate amplitude). For this reason, filtering is not a necessary requirement. The VNA sampled the data at the frequency of 30 for 2-5 minutes and transmit to PC for analysis. A 15-second Hann Window was applied the to window the respiration signal, and then process this obtained signal using Fast Fourier Transform, finally find the central frequency in the range from $0.1 \mathrm{~Hz}$ to $0.7 \mathrm{~Hz}$, (the maximum breathing rate). After finishing these step, the window moves on 1secon for another analysis. Every 8 results were calculated to find the heart rate signal. [14] the Hann window can effectively reduce the frequency leaking compared to the rectangular window.

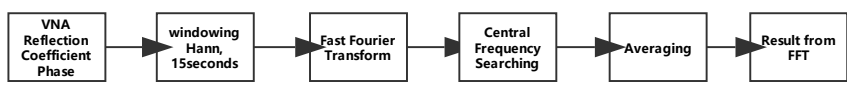

Fig. 5. Respiration rate alogrithm using FFT and time domain

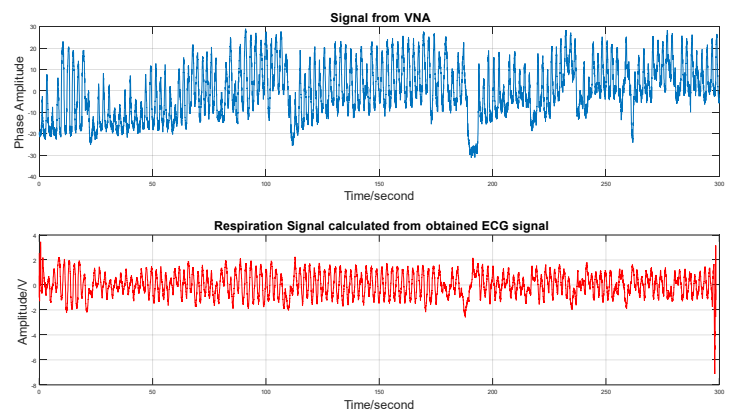

Fig. 6. Comparison between onbody respiration signal and breathing signal from ECG device. The breathing signal from commercial device was calculated using software alogrithm to extract breathing signal from obtained signal.

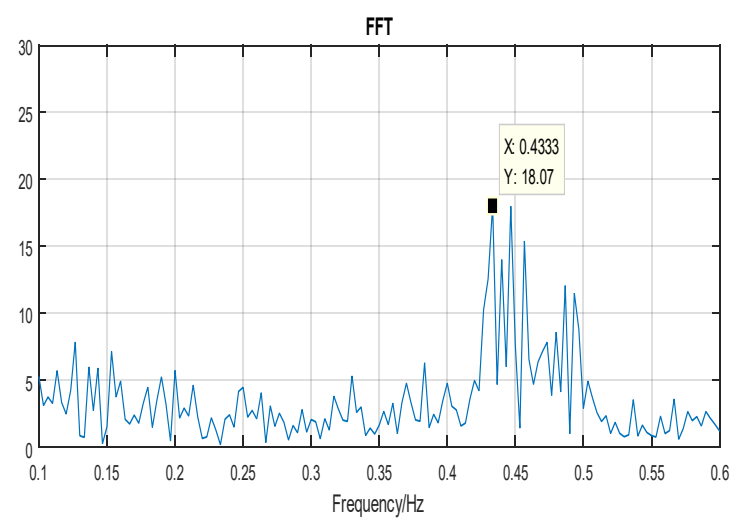

Fig. 7. Fast fourier transform of breathing signal, the central frequecy corresponding to this period is around $0.43 \mathrm{~Hz}$

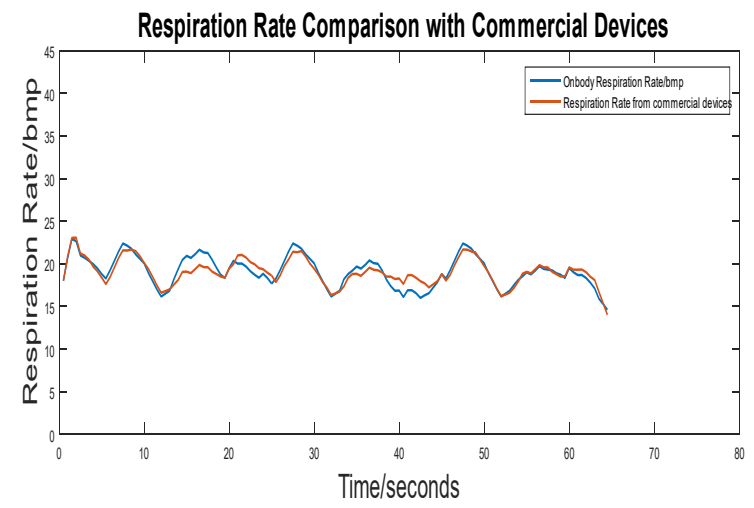

Fig. 8. Comparion between respiration rate from Onbody device signal and Respiration signal from commercial devices.

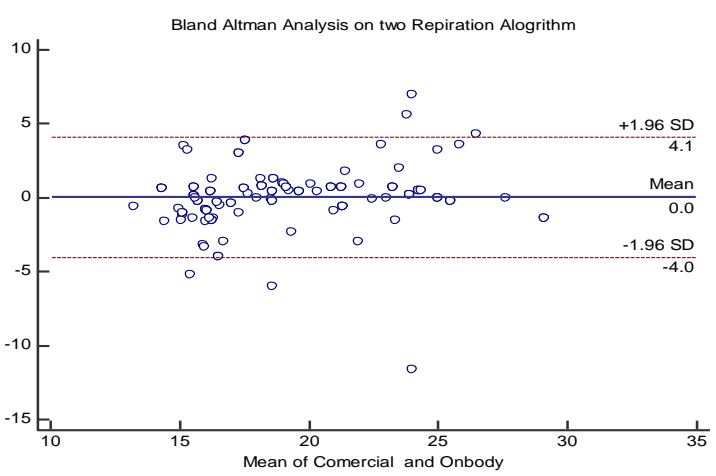

Fig. 9. Bland Atlman analysis on heart rate signal comparing to commercial device and proposed wearable method. There are 8 points out of the $95 \%$ confidence interval.

\section{Heart Rate Measurement}

For the heart rate signal, the first step involved separating the heart and breathing signal. To achieve this, the signal obtained from the vector network analyzer was passed first through a Butterworth 8th order high-pass filter with a cut-off at $0.7 \mathrm{~Hz}$ after which it was passed through a Butterworth 11th 
order lowpass filter with a cut off at $3 \mathrm{~Hz}$. After filtering, over a 10 second Hann window, the heart rate was calculated using fast Fourier transform. After the FFT, the maximum point in the range from $0.7-2.2 \mathrm{~Hz}$ was chosen as the Heart rate frequency. The reason of choosing the Han window is because it could effectively reduce the frequency leak comparing to the rectangular window. Then this 10 -second window is moved forward by one second and the same procedure applied and averaging in every 8 windows, which is a universe algorithm in commercial devices. This continues up to the end of the signal sequence. From this sample data diagram, the heart rate is $1.4 \mathrm{~Hz}(84 \mathrm{bmp})$.

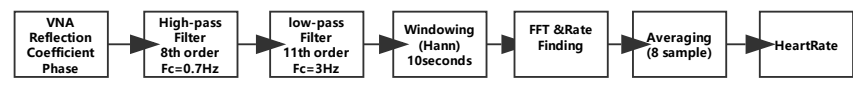

Fig. 10. Block diagrams showing the signal processing algorithm for calculating heartrate from VNA signal

Fig.12 show the signal comparison between On-body heartrate signal from Doppler radar and the ECG signal from commercial devices. Good agreement can be observed from the comparison in the second graph which representing the on-body signal as the red dashed line. In the FFT plot, the central frequency found in $0.7 \mathrm{~Hz}-2.2 \mathrm{~Hz}$ is $1.4 \mathrm{~Hz}$ (the heartrate is 84bmp). Satisfied golden standard (commercial ECG algorithm) correlation with on-body signal measurement was shown in Fig.14. For the agreement evaluation, the Bland Altman method in Fig. 15 show 8 points out of 129 sample point in total was exceed the confidence interval. The upper and low limit is 0.99 and -1.51 respectively.

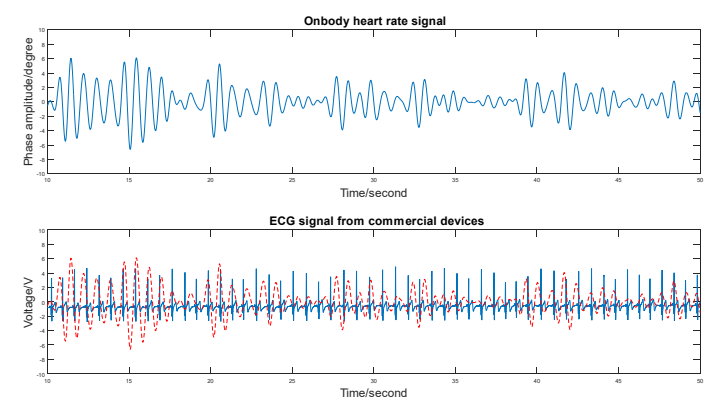

Fig. 11. Comparion between Signal from Onbody signal and heart rate signal from commercial ECG devices BMD101

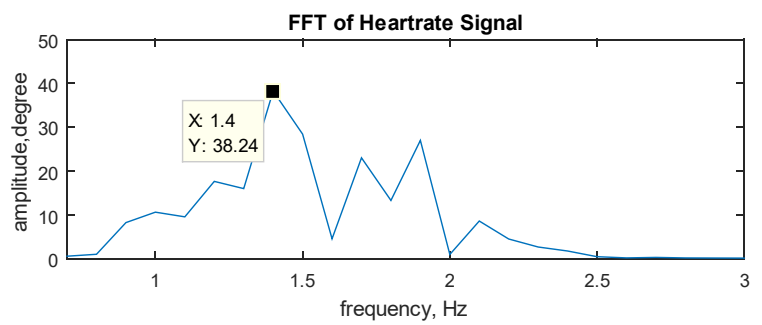

Fig. 12. The FFT result using sample, the central frequecy is $1.4 \mathrm{~Hz}$ indicating the heartrate in the time interval is $84 \mathrm{bmp}$.

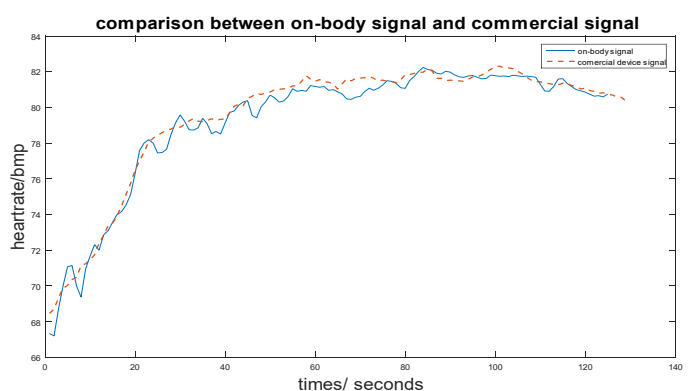

Fig. 13. Comparion between heart rate from Onbody signal and heart rate signal from commercial ECG devices BMD101

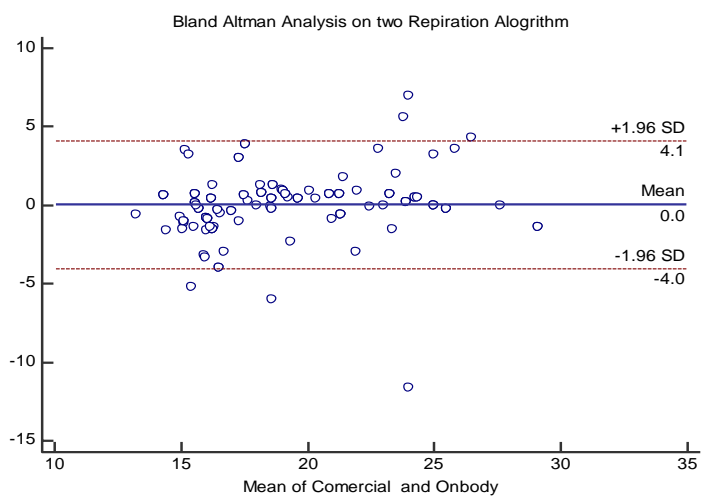

Fig. 14. Bland Atlman analysis on heart rate signal comparing to commercial device and proposed wearable method. There are 8 points out of the $95 \%$ confidence interval.

\section{CONCLUSION}

In this experiment, the result obtained from current noninvasive techniques at $2.45 \mathrm{GHz}$ can reach a satisfied result both in heart rate signal and breathing signal compared with the standard commercial device. This newly developed method can be used for measurement such as the heart rate variability (HRV) A simple human chest model has been developed for further experiment and by using this model the theoretical feasibility of on-body communication has been verified.

\section{ACKNOWLEDGMENT}

I would like to express my gratitude to my family, my friends for their support and valuable suggestions. I am also grateful to Glasgow College, UESTC, for providing me wonderful opportunity for making the project completion.

\section{REFERENCES}

[1] United Nations, Department of Economic and Social Affairs (Population Division), "World population ageing 2013," New York, Tech. Rep., 2013.

[2] R. Saxby, "How silicon will transform healthcare," in Proc. 3rd IET Int. Conf. Medical Electrical Devices Technology, Jan. 2007, pp. 135-150

[3] Khan, I.; Hall, P.S.; Serra, A.A.; Guraliuc, A.R.; Nepa, P.; "Diversity Performance Analysis for On-Body Communication Channels at 2.45 GHz," Antennas and Propagation, IEEE Transactions on , vol.57, no.4, pp.956-963, April 2009 
[4] Abubakar Tariq, "Vital Signs Monitoring using Doppler Radar and OnBody Antennas" pp85-89,Augst 2013

[5] Gabriel S, Lau RW, Gabriel C.,' The dielectric properties of biological tissues: II. Measurements in thefrequency range $10 \mathrm{~Hz}$ to $20 \mathrm{GHz}$ ', Physics in Medicine and Biology Volume 41 Number 11, 1996, pp.22512269

[6] D. Obeid, S. Sadek, G. Zaharia and G.E Zein, "Multi-Tunable Microwave System for Touchless Heartbeat Detection and Heart rate variability Extraction," Microwave and Optical Technology Letters, vol.52, no.1, pp.192-198, Jan 2010.

[7] [7] D. Droitcour, O. Boric-Lubecke, V. M. Lubecke, J. Lin, G. T. A. Kovacs, "Range Correlation and I/Q Performance Benefits in Single-Chip Silicon Doppler Radars for Noncontact Cardiopulmonary Monitoring," IEEE Transactions on Microwave Theory and Techniques, Vol. 52, No. 3, , pp. 838- 848, March 2004.

[8] D. Obeid, S. Sadek, G. Zaharia and G.E Zein, "Multi-Tunable Microwave System for Touchless Heartbeat Detection and Heart rate variability Extraction," Microwave and Optical Technology Letters, vol.52, no.1, pp.192-198, Jan 2010.

[9] D. Droitcour, O. Boric-Lubecke, V. M. Lubecke, J. Lin, G. T. A. Kovacs, "Range Correlation and I/Q Performance Benefits in Single-Chip Silicon Doppler Radars forNoncontact Cardiopulmonary Monitoring," IEEE Transactions on Microwave Theory and Techniques, Vol. 52, No. 3, , pp. 838-848, March 2004.

[10] [13] Hiebel, M., Fundamental of Vector Network Analysis, 4th ed. Germany:Rhode \&Shwarz, 2008.

[11] Changzhan Gu; Jiang Long; Jiangtao Huangfu; Qiao, S.; Cui, W.Z.; Ma, W.; Lixin Ran; ,"An instruments-built Doppler radar for sensing vital signs," Antennas, Propagation and EM Theory, 2008. ISAPE 2008. 8th International Symposium on , vol., no., pp.1398-1401, 2-5 Nov. 2008

[12] Abubakar Tariq, "Vital Signs Monitoring using Doppler Radar and OnBody Antennas" pp135,Augst 2013. 